Article

\title{
An Automated Field Phenotyping Pipeline for Application in Grapevine Research
}

\section{Anna Kicherer ${ }^{1, *}$, Katja Herzog ${ }^{1}$, Michael Pflanz ${ }^{2,3}$, Markus Wieland ${ }^{4}$, Philipp Rüger ${ }^{5}$, Steffen Kecke ${ }^{6}$, Heiner Kuhlmann ${ }^{4}$ and Reinhard Töpfer ${ }^{1}$}

1 Julius Kühn-Institut, Federal Research Centre of Cultivated Plants, Institute for Grapevine Breeding Geilweilerhof, 76833 Siebeldingen, Germany; E-Mails: katja.herzog@jki.bund.de (K.H.); reinhard.toepfer@jki.bund.de (R.T.)

2 Julius Kühn-Institut, Federal Research Centre for Cultivated Plants, Institute for Plant Protection in Field Crops and Grassland, Messeweg 11-12, 38104 Braunschweig, Germany; E-Mail: mpflanz@atb-potsdam.de

3 Leibniz Institute for Agricultural Engineering Potsdam-Bornim, Department Horticultural Engineering, Max-Eyth-Allee 100, 14469 Potsdam, Germany

4 University of Bonn, Department of Geodesy, Institute for Geodesy and Geoinformation (IGG), Nussallee 17, 53115 Bonn, Germany; E-Mails: m.wieland@uni-bonn.de (M.W.); heiner.kuhlmann@uni-bonn.de (H.K.)

5 Geisenheim University, Department of Viticultural Engineering, Brentanostraße 9, 65366 Geisenheim, Germany; E-Mail: Philipp.Rueger@hs-gm.de

6 Julius Kühn-Institut, Federal Research Centre for Cultivated Plants, Department of Data Processing, Erwin-Baur-Str. 27, 06484 Quedlinburg, Germany; E-Mail: steffen.kecke@jki.bund.de

* Author to whom correspondence should be addressed; E-Mail: anna.kicherer@jki.bund.de; Tel.: +49-6345-41127; Fax: +49-6345-919050.

Academic Editor: Vittorio M.N. Passaro

Received: 6 January 2015 / Accepted: 15 February 2015 / Published: 26 February 2015

\begin{abstract}
Due to its perennial nature and size, the acquisition of phenotypic data in grapevine research is almost exclusively restricted to the field and done by visual estimation. This kind of evaluation procedure is limited by time, cost and the subjectivity of records. As a consequence, objectivity, automation and more precision of phenotypic data evaluation are needed to increase the number of samples, manage grapevine repositories, enable genetic research of new phenotypic traits and, therefore, increase the efficiency in plant research. In the present study, an automated field phenotyping pipeline was setup and applied in a plot
\end{abstract}


of genetic resources. The application of the PHENObot allows image acquisition from at least 250 individual grapevines per hour directly in the field without user interaction. Data management is handled by a database (IMAGEdata). The automatic image analysis tool BIVcolor (Berries in Vineyards-color) permitted the collection of precise phenotypic data of two important fruit traits, berry size and color, within a large set of plants. The application of the PHENObot represents an automated tool for high-throughput sampling of image data in the field. The automated analysis of these images facilitates the generation of objective and precise phenotypic data on a larger scale.

Keywords: robot; geoinformation; high-throughput analysis; image acquisition; plant phenotyping; grapevine breeding; Vitis vinifera

\section{Introduction}

With the fast development of genotyping methods to support grapevine breeding based on SSR (Simple Sequence Repeats) [1,2] or SNP (Single Nucleotide Polymorphism) analyses, including next generation DNA sequencing [3], genotyping efficiency has been greatly improved and costs have been reduced contemporaneously. However, plant phenotyping methods have only slowly improved during the last few decades, becoming now a major bottleneck. Therefore, the lack of sufficient phenotypic data and phenotyping methods constrains the possibility to reveal the genetics of quantitative traits, such as yield, growth and adaption to abiotic or biotic stresses. The development and implementation of high-throughput phenotyping platforms is therefore a key tool to improve the efficiency of grapevine (Vitis vinifera L. subsp. vinifera) or, more generally, plant breeding. In recent years, much effort has been made to build up such platforms, which allow the assessment of large quantities of phenotypic data under controlled environments [4-9]. Although these systems enable a detailed non-invasive plant assessment throughout the plant life cycle under controlled conditions, they neglect information about the genotype-environment interactions and do not take horticultural or viticultural plants into account. However, grapevine, for example, as a rather large perennial plant, needs to be evaluated directly in the field. Several studies of the implementation of new techniques for an improved management of vineyards in practical viticulture [10-14] have been conducted in recent years. Yield estimation is one of the most important traits in precision viticulture due to annual and spatial variations. The published studies aimed to improve yield estimation and forecasting by detecting bunches of grapes, berries [15-18] or the number of inflorescences [19] in images. Ground-based sensor data used in precision viticulture are than either recoded from a constant distance to the canopy [16,19-21], mounted to a tractor [10-12], truck crane [22] or include modified vehicles [13,15,23] equipped with global positioning systems (GPS) devices $[18,24,25]$. Another approach is the application of a field phenotyping robot. Such systems have already been introduced for application in maize [26] and small grain cereals [27]. A robot application for viticulture was suggested by Longo et al. [28]. The U-Go (Unmanned Ground Outdoor) robot was developed as a multipurpose vehicle with the aim of facilitating work during the season (harvesting, pruning, transportation of bins) [28]. Furthermore, the opportunity to be equipped with a modular remote sprayer [29] is given. Its technical specification allows remote control or autonomous motion using GPS 
waypoints [28]. Nonetheless, all of these studies focus mainly on vineyard management, site-specific information to improve crop load, water or the health status of the considered plot. In contrast, grapevine breeding aims at the phenotyping of single grapevines, whereby genetic resources and large sets of breeding material need to be screened. That implies that in one experimental field plot, each plant can be a different genotype, showing its distinct phenotype, which needs to be assessed individually with high precision. Not only the resolution of phenotypic data towards one single grapevine may differ, also the variation of traits within breeding material is considerably higher than in commercial vineyards. Important phenotypic traits in grapevine breeding are the detection of fruit parameters, e.g., the berry size and color of berries. Current assessment of phenotypes in breeding programs relies largely on visual estimations, using the $\mathrm{BBCH}$ (phenological development stages of a plant; stands for Biologische Bundesanstalt, Bundessortenamt und CHemische Industrie) scale [30] or OIV (International Organization of Vine and Wine) descriptors [31]. These systems are laborious, time-consuming and, therefore, expensive. The data obtained are subjective and can vary significantly when evaluated by different persons. The biggest limitation, however, is the needed simultaneous screening of vines from several hectares of experimental vineyards, which limits a detailed evaluation of traits to a rather small number of breeding strains. The application of non-invasive, high-throughput sensor technologies is required to increase the efficiency of grapevine breeding by increasing the phenotyping efficiency (number of plants per time), improving the quality of phenotypic data recording and reducing the error variation. Such new methods progressively increase the amount of data that needs to be handled.

First steps towards a high-throughput phenotyping pipeline in grapevine breeding have been introduced by Herzog et al. [32]. The study implemented a Prototype Image Acquisition System (PIAS) for semi-automated capturing of geo-referenced images and a semi-automated image analysis tool to phenotype berry size. An automated phenotyping platform in grapevine breeding is needed to screen for phenotypic traits on a single-plant-level in a reasonable time, unlike the application in precision farming, whereas the overall appearance of a plot or at least single areas of a plot are of greatest interest.

Here, we describe the setup of an updated and expanded phenotyping pipeline involving automated data acquisition in the field, automated data management and data analysis. The challenges of this pipeline are the combination of: (1) automated simultaneous triggering of all cameras at a predefined position in the field; (2) automated acquisition of geo-referenced images; (3) data management via a database; and (4) automated image analysis for objective and precise phenotyping of the berry size and color. Moreover, we demonstrate the application of the pipeline in the grapevine repository at Geilweilerhof.

\section{Material and Methods}

\subsection{Plant Material}

The application of the phenotyping pipeline involved 2700 grapevines representing 970 accessions from the grapevine repository at the experimental vineyards of Geilweilerhof located in Siebeldingen, Germany (N 49²1.747, E $8^{\circ} 04.678$ ). Interrow distance was $2.0 \mathrm{~m}$, and grapevine spacing was $1.0 \mathrm{~m}$. Rows were planted in a north-south direction. Colored size reference labels were fixed to the wires and used to scale the images. 


\subsection{Automated Image Acquisition}

For the automated image acquisition directly in the field, the PHENObot (Phenotyping robot) was developed [33]. This phenotyping platform consists of a chain vehicle containing a control unit and a camera-light unit in combination with an industrial computer. In order to operate in a harsh outdoor environment and to enable the transportation and navigation of the camera-light unit for the non-destructive inspection of phenotypic grapevine traits, the chain vehicle had to meet certain requirements: a lifting capability up to $250 \mathrm{~kg}$, low vibration drive at a speed between 4 to $6 \mathrm{~km} \cdot \mathrm{h}^{-1}$, an easily adjustable mounting system for the sensors, a navigation system based on GPS coordinates, the ability for path planning, as well as fulfilling safety standards [33]. For targeted image acquisition, path planning is needed for the PHENObot. Therefore, precise GPS positions of individual vines are necessary and, so, all grapevines have been surveyed. The camera-light unit used on the PHENObot consists of three monochrome cameras (AVT GT-2450; objective: CVO $8 \mathrm{~mm} ; 2448 \times 2050$ pixels), one RGB camera (AVT GT-2450C; objective: Schneider KMP-IR CINEGON $8 \mathrm{~mm}$; $2448 \times 2050$ pixels) and one NIR camera (AVT MANTA; objective: Schneider KMP-IR CINEGON 8 mm; $1388 \times 1038$ ). To enable an adequate illumination for standardized image acquisition, a lightning unit containing eight LED bars (12 LEDs; ODLW300 series; Smart vision lights, Muskegon, MI, USA) was combined with the camera unit (for the setup, see Figure 1A). The components are connected with the image acquisition computer by a fast Ethernet network (GigE). All cameras are synchronously triggered using this network, and the images are transmitted immediately to the PC. The lightning unit is triggered by one of the monochrome cameras. For configuration and monitoring of the image acquisition process, a software application (IggGeotagger.Ext) has been developed fulfilling two main tasks: the communication task handles the communication between the control unit of the PHENObot and the image acquisition computer; the image acquisition task controls the cameras and the image transport and storage. The application is also used for visualization of the images and for setting the camera parameters (screenshot in Figure 1). A single image acquisition cycle performs several steps (see Figure 2). The communication task waits for a message from the PHENObot control unit. As soon as the PHENObot has reached a predefined position, it sends a specific message containing the position, the orientation and the corresponding plant ID to the computer. Then the communication task starts the image acquisition task, which triggers all cameras, receives the images, generates the filenames for the images (plantID_camera_cameraID_datetime) and saves them to the hard drive. Additionally, the position and orientation information is written directly into the file header of the image. When the image acquisition task has finished, the communication task sends an acknowledgment message to the PHENObot control, signaling that it can move to the next position. One hundred forty grapevines have been assessed to verify the image section: (1) includes the whole bunch area of each grapevine assessed, and (2) remains the same when repeatedly approached. The PHENObot was stopped at the surveyed position of the grapevine and under the consideration of the training direction (trained to the south or north, respectively). Moreover, the 140 grapevines have been approached 4 times in a row. 


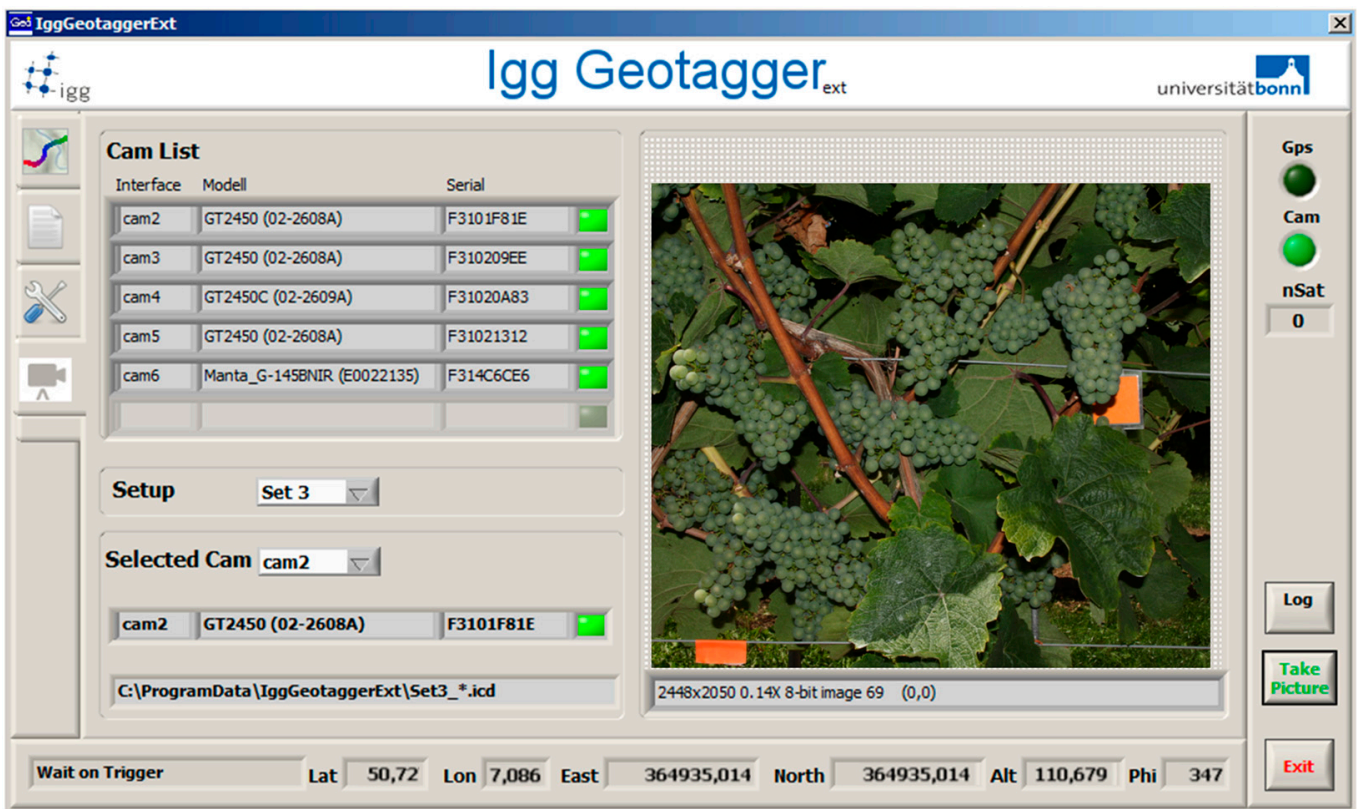

Figure 1. Graphical user interface of the IggGeotagger.Ext. The software manages the communication between the control unit of the PHENObot and the image acquisition PC, triggers the cameras and controls the image transport and storage. It is preferentially used for the visualization of captured images and for setting the camera parameters.

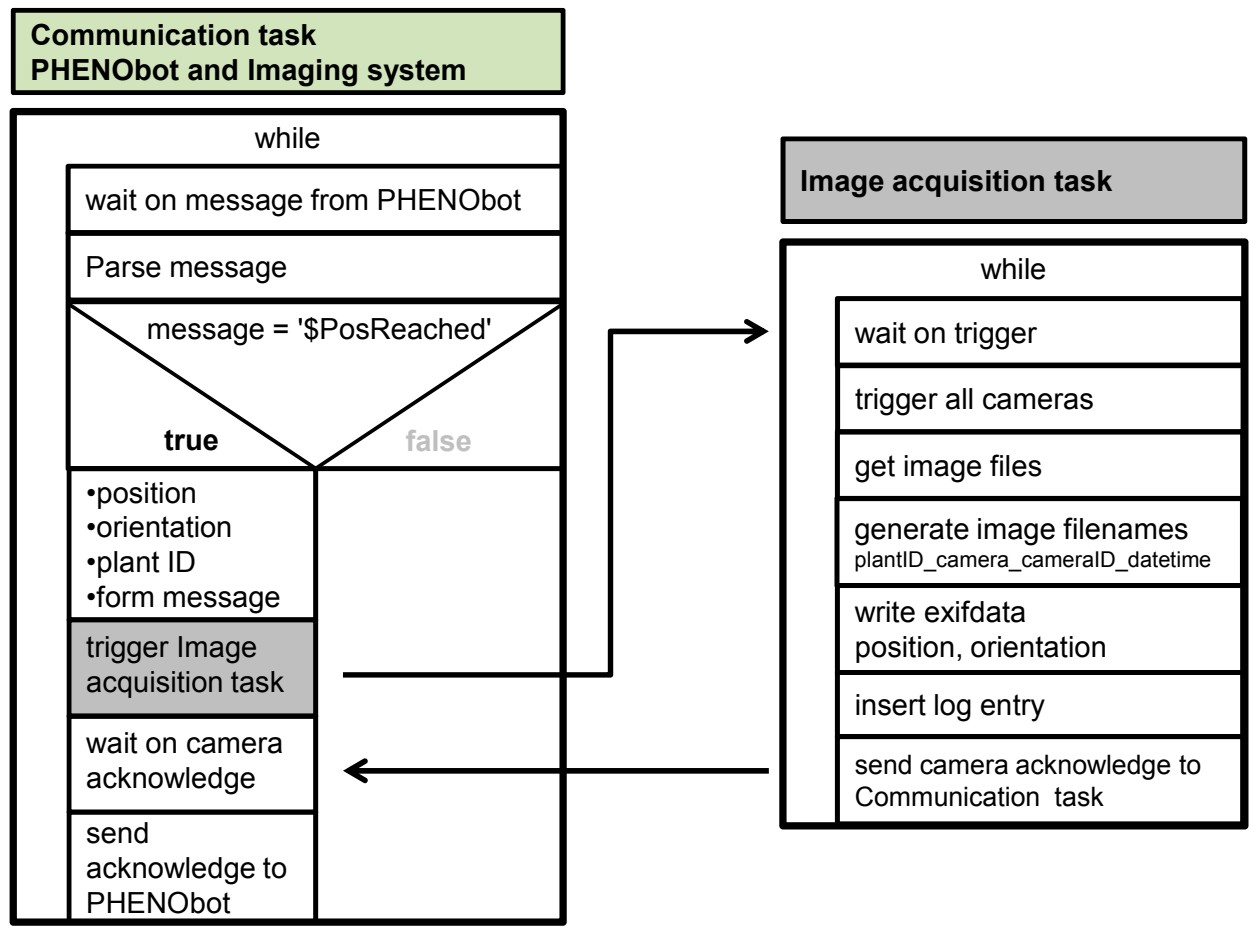

Figure 2. Communication and image acquisition task within the IggGeotagger. Ext software. The communication task handles the communication between the control unit of the PHENObot and the image acquisition PC; the image acquisition task controls the cameras and the image transport and storage. 


\subsection{Data Management}

All 2700 grapevines of the genetic repository have been surveyed using a RTK (real-time-kinematic)-GPS system (Trimble ${ }^{\circledR}$ SPS852, Geo Systems GmbH, Jena, Germany) with 2-cm accuracy. The geo-information of each grapevine and the associated plant ID is stored in the central database, PLA (Plant Location Administration) - A common management tool for experimental areas in the Julius Kühn-Institut. All images delivered by the IggGeotagger.Ext are imported into the database, IMAGEdata. Based on the image names, which contain the plant ID, every image is uniquely assigned to a single grapevine. For this assignment, the PLA is used. PLA, as well as IMAGEdata work with geographical data (UTM). The aim of IMAGEdata is to have a powerful and easy to use tool for managing the images as a basis for further evaluation. These databases can be used by modern Web 2.0 interfaces and web services. Current technologies allow safe operation and offer modern user interfaces.

\subsection{Image Analysis}

Image analysis was conducted by using the MATLAB ${ }^{\circledR}$-based tool, BIVcolor (Berries in Vineyards-color). Based on a one-class classification framework determining grapevine berry sizes, some slight modifications have been done (MATLAB 2012b and Image Processing Toolbox, The Mathworks, Natick, MA, USA) on the Berries in Vineyards (BIV) algorithm [34]. This was targeted to separately record mean RGB values of each single berry according to their color channels (RGB) and their position within the corresponding image. The data were written loop-wise into a tab-limited text file corresponding to the image file analyzed and finally stored in a SQL-database (Access 2010, Microsoft, Redmond, WA, USA). The known position of berries within a trait later on provides clustering to check berry patterns and outliers.

A set of 500 images, including 235 different accessions and $n=1,300,900$ segmented single berries, was used for color information assessment. The mean of the RGB values of all berries detected in one image were used for statistical analysis. As reference data, the berry color was assessed as five classes $(1=$ black; $2=$ red; $3=$ rose; $4=$ grey; $5=$ green $)$.

\subsection{Statistical Analysis}

Statistical analysis was conducted using the software R Version 3.1.1 (R Foundation for Statistical Computing, Vienna, Austria). Linear discriminate analysis (LDA) was performed to predict the berry color class using the RGB values as predictor variables.

\section{Results and Discussion}

\subsection{Field Application of the Phenotyping Robot}

A phenotyping pipeline has been set up and consists of the following components: (1) data acquisition; (2) data management; and (3) data analysis (Figure 3). Data acquisition was done automatically using the PHENObot. Each image was linked to one plant, respectively one genotype, without any post-processing. Applying the PHENObot image data from 2700 grapevines representing 970 grapevine accessions has been done. Automated data recording for these large set of plants was 
completed within $12 \mathrm{~h}$. The image acquisition of one grapevine took on average $15 \mathrm{~s}$. Although the camera was equipped with a lightning unit, it was impossible to take standardized images on sunny days (Figure 4). Consequently, the image acquisition in the grapevine repository was done at night due to uniform light conditions. This has also been reported to work best for images taken in commercial vineyards to estimate yield [18].

Two pre-test drives consisting of 140 grapevines have been done. The first one to ascertain the image section comprises the whole bunch zone of each grapevine assessed and the second one to make sure the same image section is captured each time a grapevine is approached. The image section was best when the stopping position of the PHENObot was shifted $25 \mathrm{~cm}$ south or north in accordance with the training direction in order to enable one to see as much of the bunch zone as possible. The 140 grapevines were approached four times, and the image section stayed the same for each grapevine and all four repetitions. The comparison of the GPS position logged at the image acquisition point for the four drives showed a difference of 1-2 cm, which is within the accuracy of the GPS system.

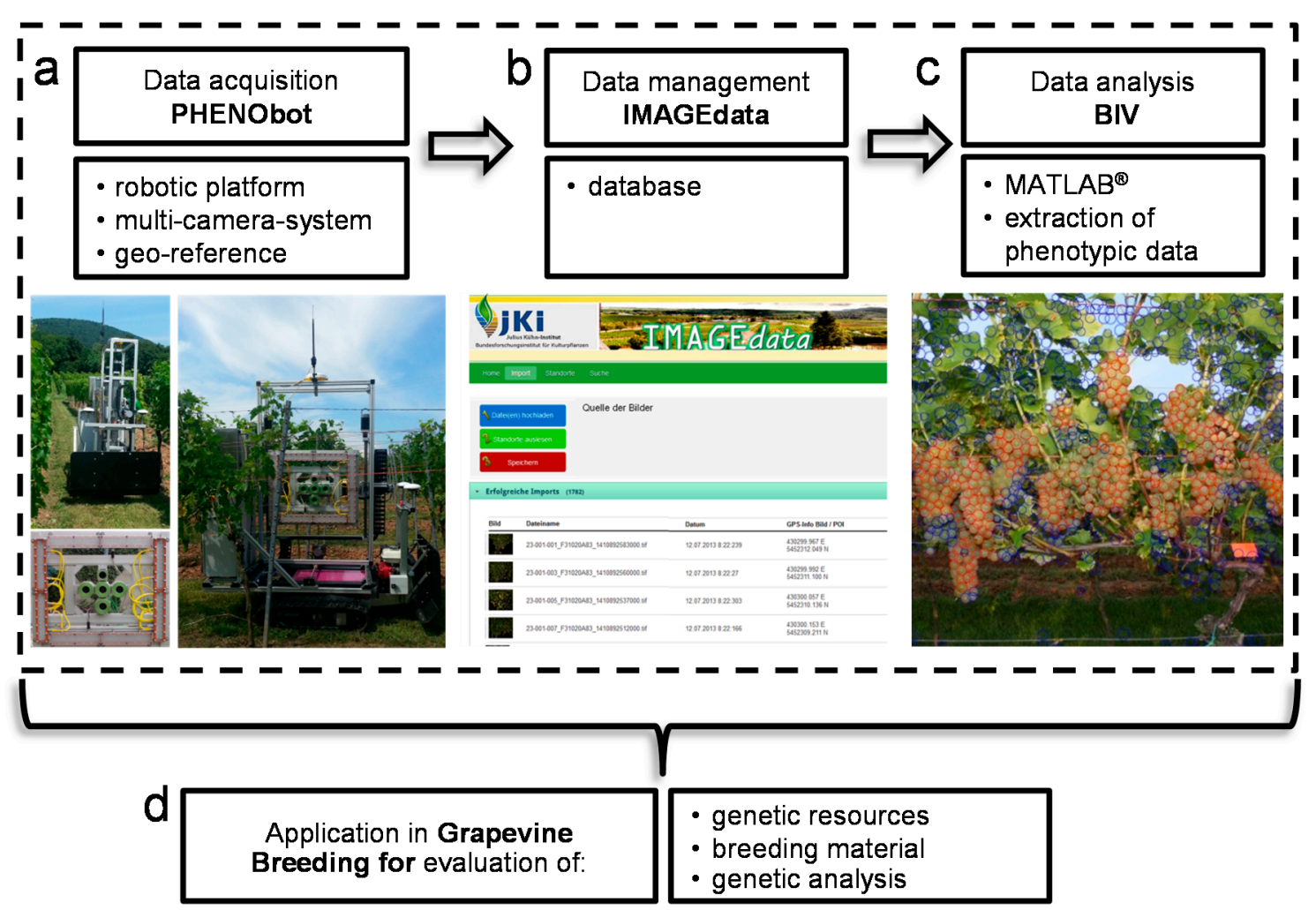

Figure 3. Phenotyping pipeline in grapevine breeding. (a) Data acquisition using the PHENObot consisting of a robotic platform, a multi-camera-system and a geo-information system; (b) data management of the sensor data is achieved by a database (IMAGEdata); (c) data analysis through the application of MATLAB ${ }^{\circledR}$-based tools, e.g., BIVcolor (Berry in Vineyards-color), to extract the phenotypic data; (d) the phenotyping pipeline was developed for application in grapevine breeding. This enables the phenotyping of large sets of plant material from genetic resources or breeding material. 

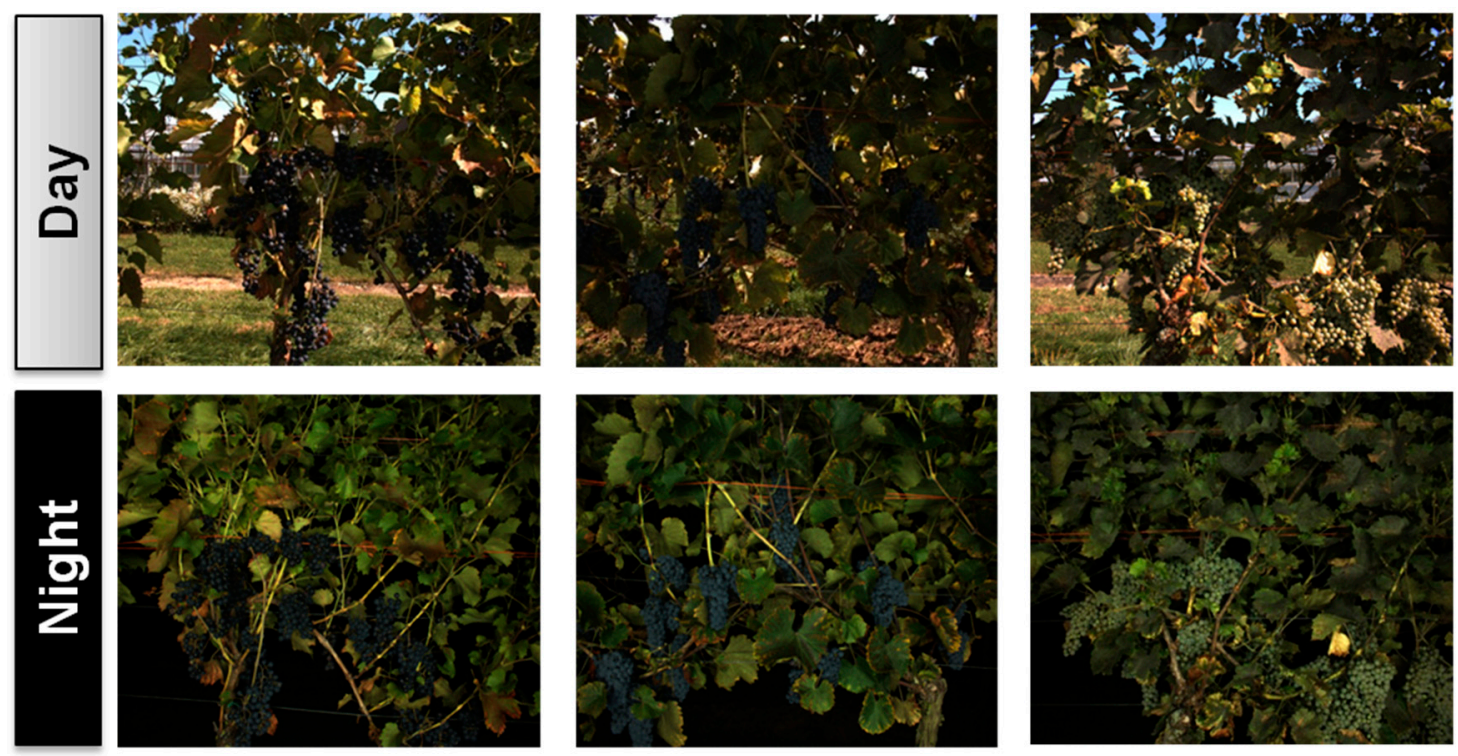

Figure 4. Comparison of images taken during the day and at night. Three examples of vines photographed on a sunny day and at night. All images were captured using the PHENObot with the lightning unit on. Image acquisition at night enables standardized conditions, which are very important for robust automated image analysis and comparable phenotyping results, e.g., with regard to the determination of berry colors.

\subsection{Image Analysis}

Images have been analyzed using the MATLAB ${ }^{\circledR}$-based tool, BIVcolor. The tool enables the automated extraction of the phenotypic traits, berry size and color. The berry size is one of the most important fruit parameters integrated for seedling selection in breeding programs. The BIVcolor evaluated berry size ranging from $9.8 \mathrm{~mm}$ to $13.9 \mathrm{~mm}$. The acquisition of the berry color is important for the characterization of genetic repositories or the phenotyping of mapping populations for genetic analysis. Initially, the color of grapes can be classified according to the presence or absence of anthocyanin in the berry skin, as either black or green. As a result of natural hybridization and human selection, the grape skin color is very diverse nowadays, ranging from green-yellow, grey, rose, red to black. The reference assessment for berry color in the set of 500 images showed a distribution of: 202 (Class $1=$ black), 200 (Class $5=$ green), 39 (Class $4=$ grey), 37 (Class $2=$ red) and 22 (Class $3=$ rose) (Figure 5a). Linear discriminant analysis (LDA) using three predictor variables (red, green and blue color values) was used to predict the class of berry color. Table 1 shows the cross-validation of the real $v s$. predicted color class. The percentage of the correct prediction of black (197 berries; 97\%) and green (178 berries; 89\%) berries was very high. Some of the green berries were predicted as grey, but in most cases, grey berries were predicted as grey ( 28 berries; $71 \%$ ). Thirteen images (59\%) visually assessed as rose berries have been predicted as red. The difference between red and rose berries can be difficult to discern no matter whether one predicts the class doing visual estimations (Figure 5a) or if one uses RGB values (Figure 5b,c). Due to the fact that RGB values of these two classes are very similar and overlapping (Figure 5b,c), it was not possible to distinguish these two classes in our study. One can clearly distinguish between black, green, grey and red/rose berries, and this is exactly what can be used for the evaluation of genetic resources and breeding material, but also for the management of grapevine 
repositories. Usually, three grapevines of one accession are planted next to each other, through the image-based color detection planting mistakes based on wrong berry color can be uncovered, for instance.
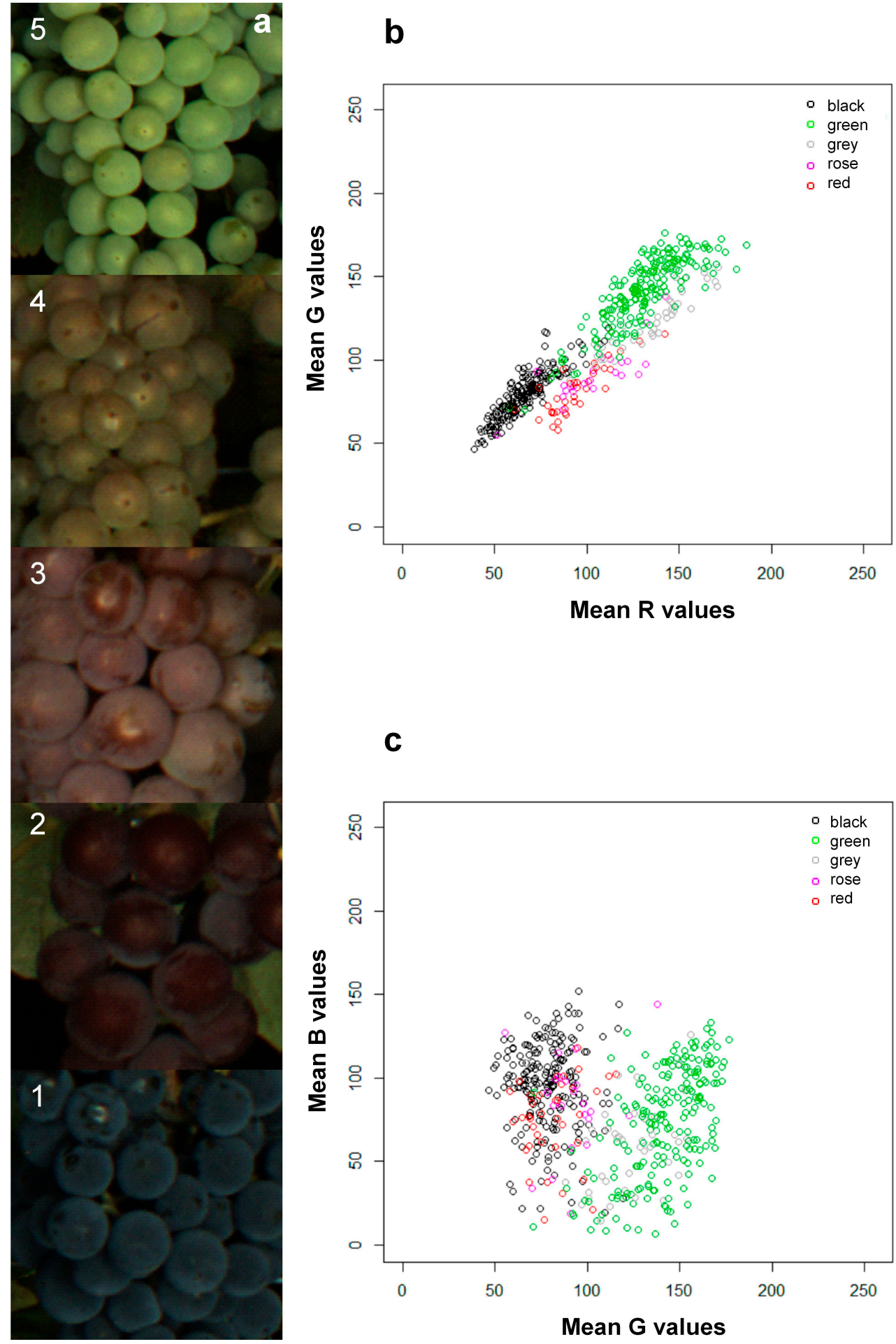

Figure 5. Distance plots of single RGB values indicating the fitness of the color model used for LDA. Prediction of berry color classes was done using the image-based detected RGB values. LDA used three parameters (red, green and blue color values) and, as the ground truth, the visually assessed berry color. (a) Berry color was visually assessed as five classes: Class 1 = black; Class 2 = red; Class 3 = rose; Class 4 = grey; Class 5 = green; (b) distance plot of $\mathrm{R}$ values vs. $\mathrm{G}$ values; (c) distance plot of $\mathrm{G}$ values vs. B values. 
Table 1. Cross-validation of the real berry color classes assessed by visual estimation and the color classes predicted with the LDA.

\begin{tabular}{cccccc}
\hline & \multicolumn{5}{c}{ Real Color Classes } \\
\hline Predicted Color Classes & Black & Green & Grey & Red & Rose \\
\hline black & 197 & 7 & 2 & 5 & 3 \\
green & 5 & 178 & 7 & 0 & 0 \\
grey & 0 & 15 & 28 & 2 & 3 \\
red & 0 & 0 & 1 & 26 & 13 \\
rose & 0 & 0 & 1 & 4 & 3 \\
\hline
\end{tabular}

From previous work presented by Roscher et al. [34], it is known that the acquisition of images in the field and automated image analysis in order to determine berry sizes is about 24-times faster compared to the application of a caliper to measure the diameter of 50 berries per grapevine. The image analysis runs automatically and needs no user interaction after starting the program. Thus, the analysis can be performed simultaneously as daily work within the common breeding program. With the extension of the $B I V$ tool [34] to $B I V$ color, we gained information about an additional phenotypic trait that can be extracted from the images without losing any time for evaluations. Another advantage is that images can always be analyzed retrospectively when new tools come along.

\subsection{Future Work}

The phenotyping pipeline has been successfully tested in grapevine breeding. So far, only the RGB images are used for automated image analysis. The camera unit consisting of five cameras (one RGB, three monochrome and one NIR camera) offers more opportunities. It enables the generation of 3D information using the monochrome cameras [32]; furthermore, it is suitable to use the NIR information for vitality indices. In addition, it is conceivable that the sensor unit of the PHENObot is going to be extended by additional sensors, like lasers, multi- or hyper-spectral sensors. There are plans to connect the IMAGEdata database with other existing databases, like VIVC (Vitis International Variety Catalogue [35]) and the European Vitis Database [36], to complete the linkage of available information.

An important stage in grapevine development is the beginning of berry ripening, namely veraison. This is the time when the berries start to soften and colored cultivars start to change their color, e.g., from green to black. It is conceivable that BIVcolor can be used to detect that date if images are taken continuously throughout the growing period.

\section{Conclusions}

A setup of a phenotyping pipeline has been introduced for grapevine breeding and to support the management of a grapevine repository. A robotic platform, the PHENObot, was built to enable the automatic image acquisition directly in the field. In order to facilitate the management of the data gained by automated image acquisition, an image database was developed. Compared to human visual assessments, a larger set of grapevines can be screened automatically, and the data revealed are objective and precise. 


\section{Acknowledgments}

We gratefully acknowledge the financial support of Projektträger Jülich and the German Federal Ministry of Education and Research (Bundesministerium für Bildung und Forschung, BMBF). This work was funded by BMBF in the framework of the projects, PHENOvines (FKZ0315968A and B) and CROP.SENSe.net (FKZ0315534). We thank Rainer Keicher and Hans-Peter Schwarz from Geisenheim University for very successful cooperation within the project PHENOvines and the development of the PHENObot vehicle.

\section{Author Contributions}

A.K., K.H. and R.T. designed the study and coordinated it. A.K. and P.R. carried out the field trail. A.K. validated the results and drafted the manuscript. M.P. extended the image analysis script, carried out the image analysis and did the LDA. M.W. and H.K. developed the software IggGeotaggerEXT. S.K. developed the database. K.H., M.P., M.W., S.K. and R.T. helped to draft the manuscript. All authors read and approved the final manuscript.

\section{Appendix}

To be able to reproduce results and conclusions, a set of 100 RGB images and the associated results from BIVcolor were deposited and are freely accessible at the data repository of the Julius Kühn-Institut, the Federal Research Centre for Cultivated Plant in Germany (doi:10.5073/jki-data.2015.1).

\section{Conflicts of Interest}

The authors declare no conflicts of interest.

\section{References}

1. Thomas, M.R.; Scott, N.S. Microsatellite repeats in grapevine reveal DNA polymorphisms when analysed as sequence-tagged sites (STSS). Theoret. Appl. Genetics 1993, 86, 985-990.

2. Eibach, R.; Zyprian, E.; Welter, L.; Töpfer, R. The use of molecular markers for pyramiding resistance genes in grapevine breeding. Vitis 2007, 46, 120-124.

3. Barba, P.; Cadle-Davidson, L.; Harriman, J.; Glaubitz, J.; Brooks, S.; Hyma, K.; Reisch, B. Grapevine powdery mildew resistance and susceptibility loci identified on a high-resolution SNP map. Theoret. Appl. Genet. 2014, 127, 73-84.

4. Granier, C.; Aguirrezabal, L.; Chenu, K.; Cookson, S.J.; Dauzat, M.; Hamard, P.; Thioux, J.J.; Rolland, G.; Bouchier-Combaud, S.; Lebaudy, A.; et al. Phenopsis, an automated platform for reproducible phenotyping of plant responses to soil water deficit in arabidopsis thaliana permitted the identification of an accession with low sensitivity to soil water deficit. New Phytol. 2006, 169, 623-635. 
5. Walter, A.; Scharr, H.; Gilmer, F.; Zierer, R.; Nagel, K.; Ernst, M.; Wiese, A.; Virnich, O.; Christ, M.; Uhlig, B.; et al. Dynamics of seedling growth acclimation towards altered light conditions can be quantified via growscreen: A setup and procedure designed for rapid optical phenotyping of different plant species. New Phytol. 2007, 174, 447-455.

6. Reuzeau, C.; Pen, J.; Frankard, V.; de Wolf, J.; Peerbolte, R.; Broekaert, W.; van Camp, W. Traitmilltm: A discovery engine for identifying yield-enhancement genes in cereals. Mol. Plant Breed. 2005, 5, 753-759.

7. Clark, R.T.; MacCurdy, R.B.; Jung, J.K.; Shaff, J.E.; McCouch, S.R.; Aneshansley, D.J.; Kochian, L.V. Three-dimensional root phenotyping with a novel imaging and software platform. Plant Physiol. 2011, 156, 455-465.

8. Hartmann, A.; Czauderna, T.; Hoffmann, R.; Stein, N.; Schreiber, F. Htpheno: An image analysis pipeline for high-throughput plant phenotyping. BMC Bioinf. 2011, 12, 148-156.

9. Bellin, D.; Peressotti, E.; Merdinoglu, D.; Wiedemann-Merdinoglu, S.; Adam-Blondon, A.F.; Cipriani, G.; Morgante, M.; Testolin, R.; di Gaspero, G. Resistance to plasmopara viticola in grapevine "bianca" is controlled by a major dominant gene causing localised necrosis at the infection site. Theor. Appl. Genet. 2009, 120, 163-176.

10. Mazzetto, F.; Calcante, A.; Mena, A.; Vercesi, A. Integration of optical and analogue sensors for monitoring canopy health and vigour in precision viticulture. Precis. Agric. 2010, 11, 636-649.

11. Llorens, J.; Gil, E.; Llop, J.; Queralto, M. Georeferenced lidar 3D vine plantation map generation. Sensors 2011, 11, 6237-6256.

12. Braun, T.; Koch, H.; Strub, O.; Zolynski, G.; Berns, K. Improving pesticide spray application in vineyards by automated analysis of the foliage distribution pattern in the leaf wall. In Commercial Vehicle Technology 2010, Proceedings of the 1st Commercial Vehicle Technology Symposium (CVT 2010), Kaiserslautern, Germany, 16-18 March 2010; Berns;, K., Schindler, C., Dreßler, K., Jörg, B., Kalmar, R., Hirth, J., Eds.; pp. 539-548.

13. Berenstein, R.; Shahar, O.B.; Shapiro, A.; Edan, Y. Grape clusters and foliage detection algorithms for autonomous selective vineyard sprayer. Intell. Serv. Robot. 2010, 3, 233-243.

14. Corbett-Davies; Sam Botterill; Tom Green, R.; Saxton, V. An expert system for automatically pruning vines. In Proceedings of the 27th Conference on Image and Vision Computing New Zealand, Dunedin, New Zealand, 26-28 November 2012; pp. 55-60.

15. Nuske, S.; Achar, S.; Bates, T.; Narasimhan, S.; Singh, S. Yield estimation in vineyards by visual grape detection. In Proceedings of the International Conference on Itelligent Robots and Systems, San Francisco, CA, USA, 25-30 September 2011; IEEE: San Francisco, CA, USA, 2011.

16. Diago, M.P.; Correa, C.; Millán, B.; Barreiro, P.; Valero, C.; Tardaguila, J. Grapevine yield and leaf area estimation using supervised classification methodology on rgb images taken under field conditions. Sensors 2012, 12, 16988-17006.

17. Liu, S.; Marden, S.; Whitty, M. Towards automated yield estimation in viticulture. In Proceedings of the Australasian Conference on Robotics and Automation, Sydney, Australia, 2-4 December 2013; UNSW: Sydney, Australia, 2013.

18. Nuske, S.; Wilshusen, K.; Achar, S.; Yoder, L.; Narasimhan, S.; Singh, S. Automated visual yield estimation in vineyards. J. Field Robot. 2014, 31, 837-860. 
19. Diago, M.P.; Sanz-Garcia, A.; Millan, B.; Blasco, J.; Tardaguila, J. Assessment of flower number per inflorescence in grapevine by image analysis under field conditions. J. Sci. Food Agric. 2014, 94, 1981-1987.

20. Fuentes, S.; Bei, R.; Pech, J.; Tyerman, S. Computational water stress indices obtained from thermal image analysis of grapevine canopies. Irrig. Sci. 2012, 30, 523-536.

21. Fuentes, S.; Poblete-Echeverría, C.; Ortega-Farias, S.; Tyerman, S.; de Bei, R. Automated estimation of leaf area index from grapevine canopies using cover photography, video and computational analysis methods. Aust. J. Grape Wine Res. 2014, 20, 465-473.

22. Möller, M.; Alchanatis, V.; Cohen, Y.; Meron, M.; Tsipris, J.; Naor, A.; Ostrovsky, V.; Sprintsin, M.; Cohen, S. Use of thermal and visible imagery for estimating crop water status of irrigated grapevine. J. Exp. Bot. 2007, 58, 827-838.

23. Calcante, A.; Mena, A.; Mazzetto, F. Evaluation of "ground sensing" optical sensors for diagnosis of plasmopara viticola on vines. Span. J. Agric. Res. 2012, 10, 619-630.

24. Mazzetto, F.; Calcante, A.; Mena, A.; Sacco, P. Test of ground-sensing devices for monitoring canopy vigour and downy mildew presence in vineyards: First qualitative traits. J. Agric. Eng. Riv. Ing. Agrar. 2011, 42, 1-9.

25. Grocholsky, B.; Nuske, S.; Aasted, M.; Achar, S.; Bates, T. A camera and laser system for automatic vine balance assessment. In Proceedings of the ASABE Annual International Meeting, Louisville, Kentucky, 7-10 August 2011; ASABE: Louisville, Kentucky, 2011.

26. Ruckelshausen, A.; Biber, P.; Dorna, M.; Gremmes, H.; Klose, R.; Linz, A.; Rahe, F.; Resch, R.; Thiel, M.; Trautz, D.; et al. Bonirob-An autonomous field robot platform for individual plant phenotyping. In Proceedings of the 7th European Conference on Precision Agriculture, Wageningen, The Netherlands, 6-8 July 2009; Academic Publishers:Wageningen, The Netherlands, 2009; pp. 841-847.

27. Busemeyer, L.; Mentrup, D.; Moller, K.; Wunder, E.; Alheit, K.; Hahn, V.; Maurer, H.P.; Reif, J.C.; Wurschum, T.; Muller, J.; et al. Breedvision-A multi-sensor platform for non-destructive field-based phenotyping in plant breeding. Sensors 2013, 13, 2830-2847.

28. Longo, D.; Pennissi, A.; Bonsignore, R.; Muscato, G.; Schillaci, G. A multifunctional tracked vehicle able to operate in vineyards using gps and laser range-finder technology. In Proceedings of the International Conference Ragusa SHWA2010, Ragusa Ibla Campus, Italy, 16-18 September 2010; pp. 487-492.

29. Longo, D.; Muscato, G.; Caruso, L.; Conti, A.; Schillaci, G. Design of a remotely operable sprayer for precision farming application. In Proceedings of the International Conference RAGUSA SHWA, Ragusa, Italy, 3-6 September 2012.

30. Lorenz, D.H.; Eichhorn, K.W.; Bleiholder, H.; Klose, R.; Meier, U.; Weber, E. Growth stages of the grapevine: Phenological growth stages of the grapevine (Vitis vinifera L. ssp. Vinifera) —Codes and descriptions according to the extended $\mathrm{BBCH}$ scale. Aust. J. Grape Wine Res. 1995, 1, $100-103$.

31. Anonymous. 2nd Edition of the Oiv Descriptor List for Grape Varieties and Vitis Species. Available online: http://www.oiv.int/oiv/info/enplubicationoiv\#grape (accessed on 6 January 2015).

32. Herzog, K.; Roscher, R.; Wieland, M.; Kicherer, A.; Läbe, T.; Förstner, W.; Kuhlmann, H.; Töpfer, R. Initial steps for high-throughput phenotyping in vineyards. Vitis 2014, 53, 1-8. 
33. Schwarz, H.P.; Rüger, P.; Kicherer, A.; Töpfer, R. Development of an autonomous driven robotic platform used for ht-phenotyping in viticulture. Mech. Eng. Lett. Szent István Univ. 2013, 10, 153-160.

34. Roscher, R.; Herzog, K.; Kunkel, A.; Kicherer, A.; Töpfer, R.; Förstner, W. Automated image analysis framework for high-throughput determination of grapevine berry sizes using conditional random fields. Comput. Electron. Agric. 2014, 100, 148-158.

35. Doligez, A.; Bouquet, A.; Danglot, Y.; Lahogue, F.; Riaz, S.; Meredith, C.P.; Edwards, K.J.; This, P. Genetic mapping of grapevine (Vitis vinifera L.) applied to the detection of QTLs for seedlessness and berry weight. Theor. Appl. Genet. 2002, 105, 780-795.

36. Doligez, A.; Audiot, E.; Baumes, R.; This, P. QTLs for muscat flavor and monoterpenic odorant content in grapevine (Vitis vinifera L.). Mol. Breed. 2006, 18, 109-125.

(C) 2015 by the authors; licensee MDPI, Basel, Switzerland. This article is an open access article distributed under the terms and conditions of the Creative Commons Attribution license (http://creativecommons.org/licenses/by/4.0/). 